\title{
Outcomes Based on Plasma Biomarkers for the Phase 3 CELESTIAL Trial of Cabozantinib versus Placebo in Advanced Hepatocellular Carcinoma
}

\author{
Lorenza Rimassa $^{a, b}$ Robin Kate Kelley ${ }^{c}$ Tim Meyer $^{d}$ Baek-Yeol Ryoo ${ }^{e}$ \\ Philippe Merle ${ }^{f}$ Joong-Won Park ${ }^{g}$ Jean-Frederic Blanc ${ }^{\text {h }}$ Ho Yeong Lim ${ }^{i}$ \\ Albert Tran ${ }^{\mathrm{j}, \mathrm{k}, \mathrm{l}}$ Yi-Wah Chan ${ }^{\mathrm{m}}$ Paul McAdam $^{\mathrm{m}}$ Evelyn Wang ${ }^{\mathrm{n}}$ \\ Ann-Lii Cheng $^{\circ}$ Anthony B. El-Khoueiry ${ }^{p}$ Ghassan K. Abou-Alfa ${ }^{q} r$
}

\begin{abstract}
${ }^{a}$ Department of Biomedical Sciences, Humanitas University, Pieve Emanuele (Milan), Italy; ${ }^{b}$ Medical Oncology and Hematology Unit, Humanitas Cancer Center, IRCCS Humanitas Research Hospital, Rozzano (Milan), Italy; ' UCSF

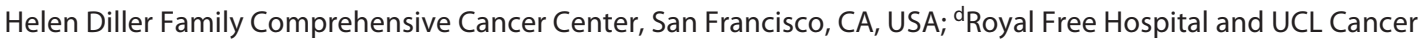
Institute, London, UK; ${ }^{\mathrm{e} A s a n}$ Medical Center, University of Ulsan College of Medicine, Seoul, Republic of Korea; ${ }^{\mathrm{f} G r o u p e m e n t ~ H o s p i t a l i e r ~ L y o n ~ N o r d, ~ L y o n, ~ F r a n c e ; ~ 9 N a t i o n a l ~ C a n c e r ~ C e n t e r, ~ G o y a n g, ~ R e p u b l i c ~ o f ~ K o r e a ; ~}{ }^{\text {hHôpital }}$ Haut-Lévêque, CHU Bordeaux, Bordeaux, France; 'Samsung Medical Center, Sungkyunkwan University School of Medicine, Seoul, Republic of Korea; JUniversité Côte d'Azur, Nice, France; ${ }^{2} \mathrm{CHU}$ de Nice, Digestive Center, Nice,

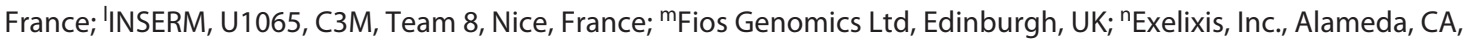
USA; ${ }^{\circ}$ National Taiwan University College of Medicine, Taipei, Taiwan; PUSC Norris Comprehensive Cancer Center, Los Angeles, CA, USA; 'Memorial Sloan Kettering Cancer Center, New York, NY, USA; 'Weill Medical College at Cornell University, New York, NY, USA
\end{abstract}

\section{Keywords}

Hepatocellular carcinoma · Plasma biomarkers · Prognostic factors · Cabozantinib

\begin{abstract}
Introduction: Cabozantinib, an inhibitor of $M E T, A X L$, and VEGF receptors, significantly improved overall survival (OS) and progression-free survival (PFS) versus placebo in patients with previously treated advanced hepatocellular carcinoma (HCC). In this exploratory analysis, outcomes were evaluated according to plasma biomarker levels. Methods: Baseline plasma levels were evaluated for MET, AXL, VEGFR2, HGF, GAS6, VEGF-A, PIGF, IL-8, EPO, ANG2, IGF-1, VEGF-C, and c-KIT for 674/707 randomized patients; and Week 4 levels were evaluated for MET, AXL, VEGFR2, HGF, GAS6, VEGF-
\end{abstract}

A, PIGF, IL-8, and EPO for 614 patients. OS and PFS were analyzed by baseline levels as dichotomized or continuous variables and by on-treatment changes at Week 4 as continuous variables; biomarkers were considered potentially prognostic if $p<0.05$ and predictive if $p<0.05$ for the interaction between treatment and the biomarker. Multivariable analyses adjusting for clinical covariates were also performed. $\boldsymbol{R} \boldsymbol{e}$ sults: In the placebo group, high levels of MET, HGF, GAS6, IL-8, and ANG2 and low levels of IGF-1 were associated with shorter OS in univariate and multivariable analyses; these associations were also observed for MET, IL-8, and ANG2 in the cabozantinib group. Hazard ratios for OS and PFS favored cabozantinib over the placebo at low and high baseline levels for all biomarkers. No baseline biomarkers were

E. Wang is no longer an employee of Exelixis.
C 2021 The Author(s).

Published by S. Karger AG, Basel

This is an Open Access article licensed under the Creative Commons Attribution-NonCommercial-4.0 International License (CC BY-NC) (http://www.karger.com/Services/OpenAccessLicense), applicable to the online version of the article only. Usage and distribution for commercial purposes requires written permission.
Correspondence to:

Lorenza Rimassa, lorenza.rimassa@ hunimed.eu 
predictive of a treatment benefit. Cabozantinib promoted pharmacodynamic changes in several biomarkers, including increases in VEGF-A, PIGF, AXL, and GAS6 levels and decreases in VEGFR2 and HGF levels; these changes were not associated with OS or PFS. Conclusion: Cabozantinib improved OS and PFS versus placebo at high and low baseline concentrations for all biomarkers analyzed. Low baseline levels of MET, HGF, GAS6, IL-8, and ANG2 and high levels of IGF-1 were identified as potential favorable prognostic biomarkers for survival in previously treated advanced HCC. Although cabozantinib promoted pharmacodynamic changes in several biomarkers, these changes were not associated with survival.

(c) 2021 The Author(s).

Published by S. Karger AG, Basel

\section{Introduction}

Hepatocellular carcinoma (HCC) is a clinically and molecularly heterogeneous disease, and this diversity has impeded the identification of prognostic plasma biomarkers associated with the clinical outcome and predictive plasma biomarkers associated with a treatment benefit for specific therapies. Alpha-fetoprotein (AFP), the most thoroughly characterized protein in this context, has been associated with the prognosis of HCC, with low serum AFP levels associated with improved survival across stages of the disease $[1,2]$. Previous studies have identified other plasma biomarkers as potential prognostic factors in advanced HCC, including proteins related to angiogenesis and/or receptor tyrosine kinase signaling such as VEGF-A, ANG2, HGF, MET, IGF-1, and IGF-2 and proteins related to inflammation such as IL- 6 and IL-8 [3-6]. Predictive biomarkers associated with response to a specific therapy have not been defined to date in HCC. Recently, a broad survey of plasma proteins identified several potentially predictive biomarkers for a survival benefit with the multitargeted receptor tyrosine kinase inhibitor (TKI) regorafenib versus placebo [6]. In addition, studies have shown a differential treatment benefit for the anti-VEGFR2 antibody ramucirumab compared with the placebo based on serum levels of AFP [7].

Cabozantinib inhibits receptor tyrosine kinases implicated in HCC progression, tumor immunosuppression, and resistance to antiangiogenic therapy, including VEGF receptors 1-3, MET, and the TAM family kinases TYRO3, AXL, and MER [8]. In the pivotal phase 3 CELESTIAL trial, cabozantinib significantly improved overall survival (OS) and progression-free survival (PFS) versus placebo in patients with advanced HCC previously treated with sorafenib and up to 2 prior systemic regimens. The median OS was 10.2 months for cabozantinib versus 8.0 months for the placebo (hazard ratio [HR] $0.76,95 \%$ confidence interval 0.63-0.92; $p=0.005)$, and the median PFS was 5.2 months versus 1.9 months (HR $0.44,95 \%$ confidence interval $0.36-0.52 ; p<0.001)$ [9].

Here, we present an exploratory analysis of OS and PFS in CELESTIAL based on plasma biomarker levels at baseline and on-treatment changes at Week 4. Biomarkers chosen for the study included cabozantinib targets (MET, AXL, VEGFR2, and c-KIT) and their ligands (HGF, GAS6, VEGF-A, PlGF, and VEGF-C) and other plasma proteins with reported prognostic significance in HCC (IL-8, EPO, ANG2, and IGF-1) [4]. Outcomes based on AFP levels in CELESTIAL have been previously reported [10] and were not included in the current analysis.

\section{Materials and Methods}

\section{Study Design and Patients}

The study design and methods for the global, randomized, placebo-controlled phase 3 CELESTIAL trial (NCT01908426) have been previously reported [9]. Eligible patients had HCC that was not amenable to curative treatment, Child-Pugh A liver function, and Eastern Cooperative Oncology Group performance status of 0 or 1 . Patients must have received prior sorafenib and could have received up to 2 prior systemic regimens for HCC, with disease progression on at least one prior regimen.

Patients were randomized 2:1 to receive cabozantinib $(60 \mathrm{mg}$ orally, once daily) or a matched placebo. Randomization was stratified by disease etiology; geographic region; and the presence of extrahepatic spread, macrovascular invasion, or both. Patients continued to receive study treatment as long as they experienced clinical benefit as judged by the investigator or until they experienced unacceptable toxicity. Treatment interruptions and dose reductions (to $40 \mathrm{mg}$ and then to $20 \mathrm{mg}$ ) were used to manage adverse events.

The study was conducted in accordance with the International Conference on Harmonization Good Clinical Practice guidelines and the principles of the Declaration of Helsinki. The Ethics Committee or institutional review board at each center approved the protocol, and all patients provided written informed consent.

\section{Endpoints and Assessments}

The primary endpoint was OS, and secondary endpoints were PFS and the objective response rate. The clinical outcome according to plasma biomarker levels was an exploratory endpoint.

Tumor response and progression were assessed every 8 weeks by the investigator according to Response Evaluation Criteria in Solid Tumors, version 1.1 [11]. The data cutoff date was June 1, 2017.

Plasma samples were collected at baseline and on treatment at Week 4 (Week 5, Day 1). Samples were analyzed for soluble biomarker levels by the Luminex assay (Assay Gate, Ijamsville, MD, USA). Baseline biomarker levels were determined for 13 proteins (MET, AXL, VEGFR2, HGF, GAS6, VEGF-A, PlGF, IL-8, EPO, 
ANG2, IGF-1, VEGF-C, and c-KIT), and Week 4 biomarker levels were determined for 9 proteins (MET, AXL, VEGFR2, HGF, GAS6, VEGF-A, PlGF, IL-8, and EPO) due to limited availability of plasma samples.

\section{Statistical Analysis}

Efficacy and safety outcomes for CELESTIAL have been previously reported [9]. The exploratory biomarker analyses reported here were not powered for statistical significance and are considered hypothesis-generating. Analyses were performed with SAS version 9.4 or $\mathrm{R}$ software version 3.5 or later.

On-treatment changes in biomarker levels were expressed as fold change at Week 4 from baseline using paired measurements for each patient. Fold changes were compared within each treatment group and between treatment groups using linear modeling.

For survival analyses, differences were tested using log-rank statistics, median durations were estimated by the Kaplan-Meier method, and HRs were estimated using Cox regression models. Where indicated, multivariable models were used, adjusting for macrovascular invasion (no or yes), extrahepatic spread (no or yes), AFP level ( $<400$ or $\geq 400 \mathrm{ng} / \mathrm{mL}$ ), Eastern Cooperative Oncology Group performance status ( 0 or $\geq 1)$, and albumin-bilirubin grade $(1$ or $\geq 2)$ [12]; these clinical covariates and cutoffs were chosen based on a multivariable analysis of pooled phase 3 studies with sorafenib [13] and univariate analyses of CELESTIAL [14]. No adjustments were made for multiple comparisons.

OS and PFS were evaluated according to baseline biomarkers, with protein levels expressed either as discrete variables dichotomized at the median of the combined treatment groups or as continuous variables. For subgroup analyses comparing cabozantinib versus placebo, baseline biomarker levels dichotomized at the median were also used. To identify potential prognostic factors, outcomes were evaluated for high versus low biomarker levels within each treatment group, with biomarker levels expressed either as dichotomized variables or continuous variables, using the log2transformed protein concentrations. Biomarkers were considered potentially prognostic if $p<0.05$ in these analyses. The association of on-treatment changes in biomarker levels with OS and PFS was evaluated using continuous analyses of the log2-transformed fold change from baseline at Week 4 . HR $<1$ indicates that longer survival was associated with higher protein levels in the dichotomized or continuous baseline analyses and with increased protein levels at Week 4 compared with baseline in the on-treatment analyses; whereas, an HR $>1$ favors lower protein levels at baseline or decreased levels at Week 4 for longer survival.

To identify potential predictive factors, the interaction between the biomarker and treatment was assessed using a Cox proportional hazards model with an interaction term. Biomarkers were considered potentially predictive if $p_{\text {interaction }}<0.05$ for the interaction between treatment and the biomarker level.

\section{Results}

As of June 1, 2017, 707 patients were randomized 2:1 to receive cabozantinib or the placebo. Baseline characteristics were generally balanced between the treatment groups [9]. At baseline, plasma samples for biomarker analysis were available for $447 / 470(95 \%)$ patients in the cabozantinib group and 227/237 (96\%) patients in the placebo group. Plasma samples at both baseline and Week 4 were available for $399(85 \%)$ patients in the cabozantinib group and 215 (91\%) patients in the placebo group.

Baseline levels of 13 biomarkers (MET, AXL, VEGFR2, HGF, GAS6, VEGF-A, PlGF, IL-8, EPO, ANG2, IGF-1, VEGF-C, and c-KIT) were tested for potential prognostic significance within each treatment group by comparing OS for high versus low dichotomized biomarkers using both univariate analyses and multivariable analyses adjusted for clinical covariates. Complementary continuous analyses were also performed. In the cabozantinib group, high levels of MET, HGF, IL-8, and ANG2 were associated with shorter OS in both univariate and multivariable dichotomized analyses $(p<0.05$; Table 1$)$. The associations of MET, IL-8, and ANG2 (but not HGF) were also observed by continuous analyses (online suppl. Table 1; see www.karger.com/doi/10.1159/000519867 for all online suppl. material), suggesting possible prognostic significance with cabozantinib. In the placebo group, high levels of MET, HGF, GAS6, IL-8, and ANG2 and low levels of IGF-1 were associated with shorter OS in both univariate and multivariable dichotomized analyses (Table 1); these results were also observed by continuous analyses (online suppl. Table 1), suggesting possible prognostic significance with the placebo. Other biomarkers were associated with OS in univariate dichotomized and continuous analyses but not when adjusted for clinical covariates; these included AXL, GAS6, EPO, and IGF-1 in the cabozantinib group and VEGF-A in the placebo group (Table 1; online suppl. Table 1).

The association between PFS and baseline biomarker levels was also evaluated using dichotomized and continuous analyses, both unadjusted and adjusted for clinical covariates. The only biomarker in either treatment group that was consistently associated with PFS using the 4 different approaches was ANG2 in the cabozantinib group, in which high baseline levels were associated with shorter PFS (online suppl. Tables 2, 3). High HGF in the cabozantinib group and high MET in the placebo group were associated with shorter PFS in the unadjusted and adjusted dichotomized analyses (online suppl. Table 2), but these results were not observed in the continuous analyses (online suppl. Table 3).

OS and PFS were also evaluated for cabozantinib versus placebo in subgroups defined by baseline biomarker levels dichotomized at the median. HRs favored cabozantinib over the placebo for both OS and PFS at low and high levels for all biomarkers analyzed (Fig. 1). Among all 
Table 1. OS within each treatment group comparing high versus low baseline biomarker levels

\begin{tabular}{|c|c|c|c|c|c|c|c|c|}
\hline \multirow{2}{*}{$\begin{array}{l}\text { Plasma } \\
\text { biomarker }\end{array}$} & \multicolumn{2}{|l|}{ univariate } & \multicolumn{2}{|c|}{$\begin{array}{l}\text { multivariable adjusted for } \\
\text { clinical covariates }\end{array}$} & \multicolumn{2}{|l|}{ univariate } & \multicolumn{2}{|c|}{$\begin{array}{l}\text { multivariable adjusted for } \\
\text { clinical covariates }\end{array}$} \\
\hline & $\mathrm{HR}(95 \% \mathrm{Cl})$ & $p$ value & $\mathrm{HR}(95 \% \mathrm{Cl})$ & $p$ value & $\mathrm{HR}(95 \% \mathrm{Cl})$ & $p$ value & $\mathrm{HR}(95 \% \mathrm{Cl})$ & $p$ value \\
\hline MET & $1.64(1.31-2.06)$ & $<0.001$ & $1.32(1.04-1.68)$ & 0.024 & $1.63(1.19-2.23)$ & 0.002 & $1.74(1.25-2.41)$ & 0.001 \\
\hline HGF & $1.73(1.38-2.18)$ & $<0.001$ & $1.37(1.07-1.76)$ & 0.014 & $2.05(1.50-2.82)$ & $<0.001$ & $1.87(1.33-2.64)$ & $<0.001$ \\
\hline GAS6 & $1.60(1.27-2.01)$ & $<0.001$ & $1.00(0.77-1.30)$ & 0.994 & $1.42(1.04-1.94)$ & 0.027 & $1.41(1.00-1.98)$ & 0.048 \\
\hline VEGF-A & $1.21(0.96-1.52)$ & 0.107 & $1.15(0.91-1.45)$ & 0.238 & $1.48(1.08-2.03)$ & 0.015 & $1.33(0.97-1.85)$ & 0.081 \\
\hline PIGF & $1.09(0.86-1.38)$ & 0.476 & $0.88(0.69-1.12)$ & 0.303 & $1.30(0.94-1.78)$ & 0.112 & $1.29(0.93-1.79)$ & 0.132 \\
\hline IL-8 & $1.80(1.43-2.27)$ & $<0.001$ & $1.56(1.23-1.97)$ & $<0.001$ & $1.71(1.25-2.34)$ & 0.001 & $1.54(1.11-2.14)$ & 0.011 \\
\hline EPO & $1.52(1.21-1.91)$ & $<0.001$ & $1.24(0.98-1.57)$ & 0.075 & $1.28(0.94-1.75)$ & 0.117 & $1.35(0.98-1.87)$ & 0.069 \\
\hline
\end{tabular}

Baseline biomarker levels were dichotomized at the median of the combined treatment groups. HRs $<1$ favor high over low biomarker levels. $p$ values $<0.05$ are in bold. $\mathrm{Cl}$, confidence interval; $\mathrm{HR}$, hazard ratio; OS, overall survival.

baseline biomarkers, the lowest HR for OS was 0.65 for low AXL, and the highest HR for OS was 0.92 for high AXL; the lowest HR for PFS was 0.41 for high IGF-1, and the highest HR for PFS was 0.54 for low IGF-1.

Baseline biomarkers were evaluated for potential predictive significance by modeling the interaction between treatment and the biomarker level. No baseline biomarkers were associated with a predictive effect for OS or PFS at a significance level of 0.05 (Fig. 1). The lowest $p_{\text {interaction }}$

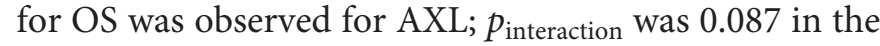
dichotomized analysis and 0.114 in the dichotomized analysis adjusted for clinical covariates (not shown). The forest plot shows the greater relative OS treatment benefit with cabozantinib at low versus high baseline AXL levels; this benefit did not reach statistical significance.

The change in plasma levels from baseline to Week 4 was determined for 9 biomarkers (MET, AXL, VEGFR2, HGF, GAS6, VEGF-A, PlGF, IL-8, and EPO). As shown in Table 2, the largest median fold decreases with cabozantinib were observed for VEGFR2 (0.60-fold change) and HGF (0.71-fold change), and the largest median fold increases were observed for PlGF (4.28-fold change) and VEGF-A (3.80-fold change). Cabozantinib also promoted smaller increases in the levels of AXL, GAS6, and EPO from baseline that were statistically different versus changes with the placebo. Although on-treatment MET levels increased in the cabozantinib group, this change was not significant compared with the increase observed with the placebo. Small but statistically significant changes from baseline were also seen for several biomarkers in the placebo group, including increases in PlGF, VEGF-A, MET, and VEGFR2 and decreases in EPO and GAS6.

The association of on-treatment changes in biomarker levels with OS and PFS was evaluated by continuous analysis based on the log2-fold change in biomarker levels at Week 4 from baseline. Increasing VEGFR2 was associated with longer OS in the cabozantinib group in univariate analysis but not when adjusted for clinical covariates (Table 3). In the placebo group, increasing levels of MET, AXL, VEGFR2, HGF, GAS6, and IL-8 were associated with shorter OS in both univariate and multivariable analyses (Table 3), suggesting potential prognostic significance. Increasing levels of AXL and HGF were also associated with shorter PFS in the placebo group in both univariate and multivariable analyses (online suppl. Table 4).

On-treatment changes in biomarker levels at Week 4 were tested for predictive significance by modeling the interaction between treatment and the biomarker level. Increases in the plasma levels of MET, AXL, VEGFR2, HGF, GAS6, and IL-8 were identified as potential predictive factors for relatively worse OS with placebo compared with cabozantinib (Table 4). No plasma biomarkers were associated with a differential treatment benefit for PFS (online suppl. Table 5). 


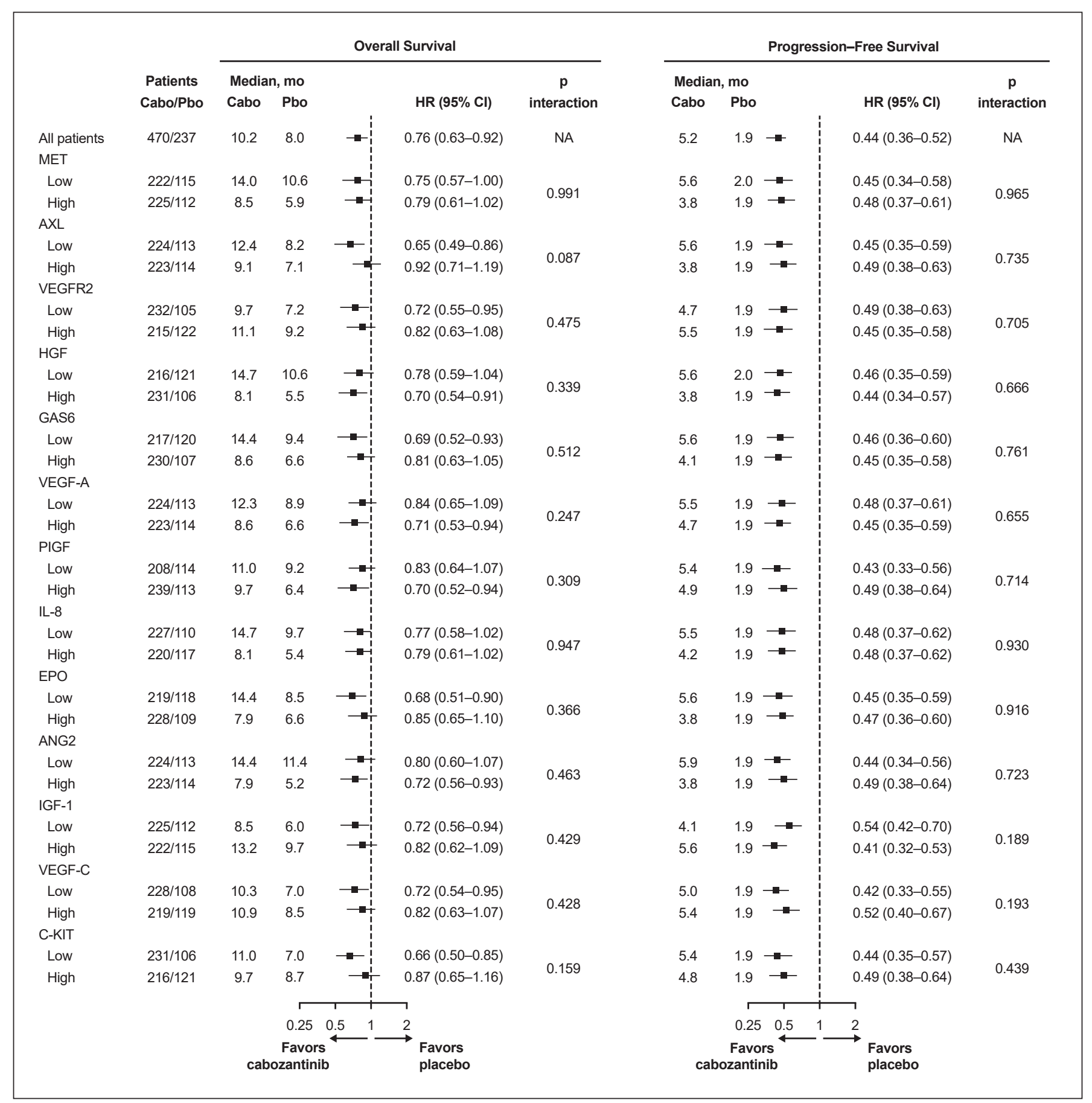

Fig. 1. Forest plots of OS and PFS for cabozantinib versus placebo by baseline biomarker levels. Baseline biomarker levels were dichotomized at the median of the combined treatment groups. $p_{\text {interaction }}$ was obtained from a separate model that included the interaction between treatment and the biomarker level. OS, overall survival; PFS, progression-free survival; CI, confidence interval; HR, hazard ratio. 
Table 2. Plasma biomarker levels at baseline and Week 4

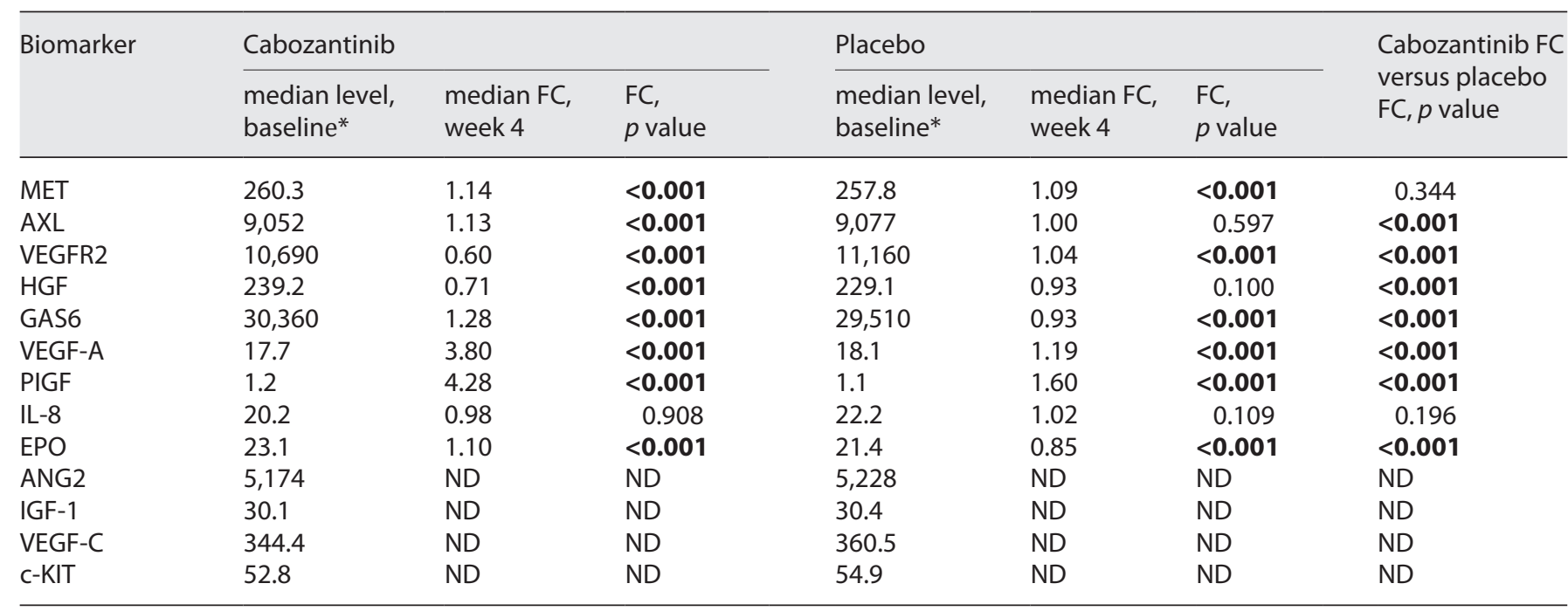

$p$ values $<0.05$ are in bold. $\mathrm{Cl}$, confidence interval; $\mathrm{FC}$, fold change; $\mathrm{HR}$, hazard ratio; ND, not determined. * Concentrations in pg/mL except MET, IGF-1, c-KIT (ng/mL) and EPO ( $\mathrm{mlU} / \mathrm{mL})$. Baseline and Week 4 measurements were paired for each patient to calculate fold changes.

Table 3. Continuous analysis of OS by on-treatment changes in biomarker levels

\begin{tabular}{|c|c|c|c|c|c|c|c|c|}
\hline \multirow{2}{*}{$\begin{array}{l}\text { Plasma } \\
\text { biomarker }\end{array}$} & \multicolumn{4}{|l|}{ Cabozantinib } & \multicolumn{4}{|l|}{ Placebo } \\
\hline & $\mathrm{HR}(95 \% \mathrm{Cl})$ & $p$ value & HR (95\% Cl) & $p$ value & HR (95\% Cl) & $p$ value & $\mathrm{HR}(95 \% \mathrm{Cl})$ & $p$ value \\
\hline MET & $1.24(0.85-1.80)$ & 0.266 & $1.37(0.93-2.03)$ & 0.115 & $3.13(1.85-5.30)$ & $<0.001$ & $2.42(1.42-4.13)$ & 0.001 \\
\hline HGF & $0.83(0.65-1.06)$ & 0.135 & $0.96(0.76-1.22)$ & 0.743 & $2.39(1.76-3.25)$ & $<0.001$ & $2.77(1.97-3.89)$ & $<0.001$ \\
\hline GAS6 & $0.82(0.62-1.08)$ & 0.153 & 1.01 (0.77-1.33) & 0.927 & $1.66(1.11-2.50)$ & 0.015 & $1.84(1.22-2.79)$ & 0.004 \\
\hline VEGF-A & $1.07(0.99-1.17)$ & 0.098 & $1.05(0.97-1.15)$ & 0.234 & $1.06(0.90-1.23)$ & 0.497 & $1.12(0.95-1.32)$ & 0.187 \\
\hline PIGF & $0.99(0.90-1.08)$ & 0.798 & $0.98(0.89-1.08)$ & 0.744 & $1.02(0.88-1.19)$ & 0.755 & $1.00(0.86-1.17)$ & 0.982 \\
\hline IL-8 & $0.88(0.75-1.04)$ & 0.134 & $0.90(0.76-1.06)$ & 0.220 & $1.52(1.22-1.90)$ & $<0.001$ & $1.47(1.17-1.85)$ & 0.001 \\
\hline EPO & $0.94(0.79-1.12)$ & 0.507 & $1.02(0.86-1.20)$ & 0.860 & $1.13(0.87-1.46)$ & 0.373 & $1.25(0.94-1.66)$ & 0.119 \\
\hline
\end{tabular}

Continuous analysis of the log2-transformed fold change in biomarker levels at Week 4 from baseline. $\mathrm{HR}<1$ indicates longer OS with increased protein levels at Week 4 compared with baseline. $p$ values $<0.05$ are in bold. $\mathrm{Cl}$, confidence interval; $\mathrm{HR}$, hazard ratio; OS, overall survival.

\section{Discussion}

The phase 3 CELESTIAL trial evaluated the efficacy and safety of cabozantinib compared with the placebo in patients with advanced HCC previously treated with sorafenib and demonstrated significantly improved OS and PFS with cabozantinib versus placebo [9]. Consistent with the results of the primary analysis, subgroup analyses based on baseline plasma biomarker levels showed improved OS and PFS with cabozantinib versus placebo at high and low concentrations for all biomarkers analyzed.

A number of baseline plasma biomarkers in the placebo group were identified as potential favorable prog- 
Table 4. Continuous analysis of OS: interaction between treatment and changes in biomarker levels

\begin{tabular}{llllllr}
\hline \multirow{2}{*}{$\begin{array}{lllll}\text { Plasma } \\
\text { biomarker }\end{array}$} & \multicolumn{2}{l}{ Unadjusted } & & \multicolumn{2}{l}{ Adjusted for clinical covariates } \\
\cline { 2 - 3 } & $\mathrm{HR}_{\text {interaction }}(95 \% \mathrm{Cl})$ & $p_{\text {interaction }}$ & & & $\mathrm{HR}_{\text {interaction }}(95 \% \mathrm{Cl})$ & $p_{\text {interaction }}$ \\
\hline MET & $0.35(0.18-0.67)$ & $\mathbf{0 . 0 0 1}$ & & $0.49(0.26-0.93)$ & $\mathbf{0 . 0 2 9}$ \\
AXL & $0.24(0.11-0.54)$ & $<\mathbf{0 . 0 0 1}$ & & $0.35(0.16-0.78)$ & $\mathbf{0 . 0 1 0}$ \\
VEGFR2 & $0.34(0.17-0.71)$ & $\mathbf{0 . 0 0 4}$ & & $0.28(0.13-0.63)$ & $\mathbf{0 . 0 0 2}$ \\
HGF & $0.33(0.22-0.48)$ & $<\mathbf{0 . 0 0 1}$ & & $0.32(0.21-0.47)$ & $<\mathbf{0 . 0 0 1}$ \\
GAS6 & $0.46(0.28-0.75)$ & $\mathbf{0 . 0 0 2}$ & & $0.51(0.31-0.82)$ & $\mathbf{0 . 0 0 6}$ \\
VEGF-A & $1.03(0.87-1.24)$ & 0.711 & & $0.95(0.79-1.14)$ & 0.581 \\
PIGF & $0.99(0.83-1.19)$ & 0.950 & & $1.02(0.86-1.22)$ & 0.807 \\
IL-8 & $0.57(0.43-0.74)$ & $<\mathbf{0 . 0 0 1}$ & & $0.60(0.46-0.80)$ & $<\mathbf{0 . 0 0 1}$ \\
EPO & $0.83(0.61-1.15)$ & 0.262 & & $0.79(0.57-1.10)$ & 0.160 \\
\hline
\end{tabular}

Continuous analysis of the log2-transformed fold change in biomarker levels at Week 4 from baseline using a model that includes the interaction between treatment and the biomarker level. HR $<1$ indicates differential treatment benefit for cabozantinib versus placebo with on-treatment increase in biomarker levels. $p$ values $<0.05$ are in bold. $\mathrm{Cl}$, confidence interval; $\mathrm{HR}$, hazard ratio; OS, overall survival. nostic biomarkers for OS, including low baseline levels of MET, HGF, GAS6, IL-8, and ANG2 and high levels of IGF-1. Among these, low baseline levels of MET, IL-8, and ANG2 were also potentially prognostic in the cabozantinib group. Several of these prognostic factors have also been identified in previous clinical studies in patients with advanced HCC including MET $[5,6], \operatorname{ANG} 2[3,6]$, HGF [5], IGF-1 [15], and IL-8 [6, 16], providing further support for their significance. Pro-inflammatory cytokines, including IL-8, have been linked to the pathogenesis of HCC and are negatively associated with clinical outcomes $[16,17]$. However, none of the baseline biomarkers were found to be predictive of a treatment benefit with cabozantinib for OS or PFS. Most previous biomarker studies in HCC also failed to identify predictive factors for treatment benefit with the exception of a broad survey of plasma proteins done for the phase 3 regorafenib trial in second-line HCC [6].

Prior studies indicate that AXL plays a role in resistance to antiangiogenic therapy, including sorafenib [18, 19]. In an integrated analysis, a small cohort of patients with HCC who were treated with sorafenib and had high baseline serum levels of soluble AXL had shorter duration of sorafenib treatment and OS versus those with low baseline levels [19]. AXL was also overexpressed in HCC cell lines and correlated with epithelial-to-mesenchymal transition and sorafenib resistance. In the current study, low baseline levels of AXL were favored over high levels for OS outcomes in both cabozantinib and placebo arms in univariate analysis, but this was not maintained in multivariable analysis.
Cabozantinib promoted an increase in the plasma levels of VEGF-A and PIGF and a decrease in the level of VEGFR2, which are well-characterized pharmacodynamic effects of VEGFR TKIs [20] that have been previously reported with cabozantinib in other tumor types $[21,22]$. Pharmacodynamic changes were also observed in components of other signaling pathways inhibited by cabozantinib, including an increase in AXL and its ligand GAS6 and a decrease in HGF with cabozantinib compared with the placebo. Although MET levels also increased in the cabozantinib group, this change was not significant compared with the increase also seen in the placebo group. Cabozantinib-promoted increases in AXL and GAS6 have also been reported in a phase 2 study in metastatic castration-resistant prostate cancer [19, 21]; however, effects on MET and HGF have been less pronounced and less consistent across trials, perhaps due to differences in tumor types or time points assessed [21, 22].

Although cabozantinib promoted significant pharmacodynamic effects, on-treatment changes in plasma cytokine levels were not associated with OS or PFS with cabozantinib. Likewise, extensive investigations of other VEGF signaling inhibitors have failed to find convincing evidence for a correlation between pharmacodynamic changes in plasma cytokines and angiogenic proteins and efficacy $[20,23,24]$. Consistent with these results, preclinical studies with sunitinib in nontumor-bearing mice suggest that these pharmacodynamic changes induced by VEGFR TKIs may be a systemic response to treatment rather than a tumor-dependent response [25]. In con-
44

Liver Cancer 2022;11:38-47 DOI: $10.1159 / 000519867$
Rimassa et al. 
trast, decreased levels of serum AFP have been associated with improved OS across stages of HCC, including in advanced HCC with sorafenib, ramucirumab, regorafenib, cabozantinib, and the combination of atezolizumab and bevacizumab [10, 26-32].

Despite relatively modest on-study changes in biomarker levels in the placebo group, increasing levels of MET, AXL, VEGFR2, HGF, GAS6, and IL-8 were identified as possible negative prognostic factors for OS with the placebo and as potential predictive factors for a differential treatment benefit for OS with cabozantinib versus placebo. Among these plasma proteins, high baseline levels of MET, HGF, GAS6, and IL-8 were also associated with negative prognosis for OS in the placebo group. Although these analyses for predictive factors met the statistical significance level, their clinical utility is limited given that the results were driven by numerically small on-treatment changes in the placebo group, which nonetheless showed a statistically significant association with OS. In addition, the result was based on a continuous analysis rather than a defined cutoff for biomarker levels, thereby limiting practical utility.

The current results were based on a large, successful phase 3 trial that included a placebo control arm, with plasma samples available for the majority of patients. Furthermore, baseline biomarkers were judged as potentially prognostic only when the results were observed using 4 different approaches, dichotomized and continuous analyses both unadjusted and adjusted for clinical covariates. However, the study has several important limitations. This exploratory analysis was done post hoc, and the trial was not powered to test the correlation of biomarkers with outcomes. The study population size was chosen to assess main effects only, not to test interactions which would require a substantially larger sample size to evaluate predictive effects at the significance level of 0.05 used here [33]. Additionally, no corrections were made for multiple comparisons. Furthermore, the pleotropic and redundant nature of the signaling pathways in the tumor microenvironment of the biomarkers tested, along with the nontumor sources of the biomarkers, may confound the relationship between biomarker levels and outcomes. The study focused on plasma samples which are easier to obtain and therefore facilitate evaluation of biomarker changes over time. However, tumor biopsies may provide more information on the local tumor environment, although lack of correlation with tumor MET and epithelial-to-mesenchymal transition marker expression with outcomes has also been observed, highlighting the complexities of tumor sampling and of se- lecting the most appropriate assay for each marker [34, 35]. Other caveats include that on-treatment changes were evaluated only at Week 4 and that PFS differences in the placebo group were difficult to assess because many patients had progressed at the time of the first tumor assessment at Week 8. Also, patients who had a dose hold or reduction prior to Week 4 were included in the analysis. As this was an early time point, the proportion of patients who had a dose hold or reduction would be more limited than at later time points. Furthermore, because cabozantinib has a plasma half-life of $\sim 99 \mathrm{~h}$ [36], the influence of these patients on the overall results should be less pronounced than for a drug with a short half-life.

In conclusion, low baseline levels of MET, HGF, GAS6, IL-8, and ANG2 and high levels of IGF-1 were identified as potential favorable prognostic biomarkers for OS in patients with previously treated advanced HCC. Consistent with the results of the primary analysis, cabozantinib improved OS and PFS compared with the placebo at high and low baseline concentrations for all biomarkers analyzed, and no baseline biomarkers were found to be predictive of a treatment benefit for OS or PFS. Overall, the results support the use of cabozantinib in patients with HCC previously treated with sorafenib, irrespective of plasma biomarker levels. Future studies, including the combination trial of cabozantinib and atezolizumab in first-line HCC [37], will explore both circulating and tumor biomarkers related to angiogenic signaling, inflammation, and immune-cell function.

\section{Acknowledgments}

We thank the patients, their families, the investigators, the site staff, and the trial teams; David W. Markby (Exelixis) for medical writing assistance; and Michael Raffin (Fishawack Communications) for editorial assistance, which was funded by Exelixis. T. Meyer is partly funded by the NIHR UCLH Biomedical Research Centre.

\section{Statement of Ethics}

The study (NCT01908426) was conducted in accordance with the International Conference on Harmonization Good Clinical Practice guidelines and the principles of the Declaration of Helsinki. The Ethics Committee or institutional review board at each center approved the study protocol and amendments. All patients provided written informed consent. Full list of study locations can be found at the CELESTIAL Study Record at https://clinicaltrials. gov/ct2/show/NCT01908426. 


\section{Conflict of Interest Statement}

G.K.A.-A. reports research grants from Acta Biologica, Agios, AstraZeneca, Bayer, Beigene, Berry Genomics, BMS, Casi, Celgene, Exelixis, Genentech/Roche, Halozyme, Incyte, MabVax, Puma, QED, Sillajen, and Yiviva and consulting fees from Agios, AstraZeneca, Autem, Bayer, Beigene, Berry Genomics, Celgene, CytomX, Debio, Eisai, Lilly, Exelixis, Flatiron, Genentech/Roche, Gilead, Helio, Incyte, Ipsen, Loxo, Merck, MINA, Polaris, QED, Redhill, Silenseed, Sillajen, Sobi, Therabionics, Twoxar, Vector, and Yiviva. J.-F.B. reports personal fees from Ipsen, Bayer, Eisai, Roche, AstraZeneca, and BMS. Y.-W.C. reports payment to employer from Exelixis for statistical analysis during the conduct of the study. A.L.C. reports grant and nonfinancial support from Exelixis during the conduct of the study, including support of the parent study and funding of editorial support; honoraria and advisory/consulting fees from AstraZeneca, BMS, Exelixis, Ipsen, Bayer, and Merck; honoraria, advisory/consulting fees, and speaker bureau fees from Eisai, Novartis, and Ono Pharmaceutical; honoraria, advisory/consulting fees, and travel and accommodation expenses from Genentech/Roche and IQVIA; speaker bureau fees and travel and accommodation expenses from Bayer Yakuhin; and speaker bureau fees from Amgen Taiwan; A.L.C. is an editorial board member of Liver Cancer. A.E.-K. reports personal fees and nonfinancial publication support from Exelixis during the conduct of the study; personal fees from Genentech/Roche, BMS, Eisai, and Merck; grants and personal fees from AstraZeneca; personal fees from Bayer, Gilead, QED, Agenus, ABL Bio, Pieris, Cytomx, and EMD Serono; and grants from Astex. R.K.K. reports research funding grants to institution for conduct of clinical trials from Exelixis; research funding grants to institution for conduct of clinical trials and Steering Committee membership from Agios, AstraZeneca, BMS, and Merck; research funding grants to institution for conduct of clinical trials from Bayer, Lilly, EMD Serono, Novartis, QED, and Taiho; IDMC membership and advisory board fees from Genentech/Roche; personal fees for travel support and advisory board participation from Ipsen; and advisory board fees from Gilead and Exact Sciences. H.Y.L. reports no conflicts of interest. P. McAdam reports payment to employer from Exelixis for statistical analysis during the conduct of the study. P. Merle reports advisory board fees from Roche, Bayer, Lilly, Merck, Eisai, and AstraZeneca and grants and advisory board fees from Ipsen and Genosciences. T.M. reports consultancy fees from AstraZeneca, Bayer, Eisai, Ipsen, and Roche and research grants from BTG and
Bayer. J.-W.P. reports grants from Ipsen-Exelixis, Genentech/ Roche, AstraZeneca, and Merck and personal fees from Ipsen-Exelixis, Genentech/Roche, Bayer, Eisai, and AstraZeneca. L.R. reports grants to institution for the CELESTIAL trial, medical writing assistance, and nonfinancial support from Exelixis during the conduct of the study; consulting/advisory role fees from Amgen, ArQule, AstraZeneca, Basilea, Bayer, BMS, Celgene, Eisai, Exelixis, Genenta, Hengrui, Incyte, Ipsen, IQVIA, Lilly, Merck, Nerviano Medical Sciences, Roche, Sanofi, Servier, and Zymework; honoraria/lecture fees from AbbVie, Amgen, Bayer, Eisai, Gilead, Incyte, Ipsen, Lilly, Merck Serono, Roche, and Sanofi; travel expenses from Ipsen; and research funding grants to institution from Agios, ARMO BioSciences, AstraZeneca, Beigene, Eisai, Exelixis, Fibrogen, Incyte, Ipsen, Lilly, Merck, Nerviano Medical Sciences, Roche, and Zymeworks. B.-Y.R. has nothing to disclose. E.W. reports employment and stock ownership from Exelixis.

\section{Funding Sources}

This study was sponsored by Exelixis. The authors and the sponsor were responsible for data collection and analysis.

\section{Author Contributions}

L.R., R.K.K., and G.K.A.-A. contributed to study conception and design; L.R., R.K.K., T.M., B.-Y.R., P. Merle, J.-W.P., J.-F.B., H.Y.L., A.T., E.W., A.L.C., A.B.E.-K., and G.K.A.-A. contributed to data acquisition; Y.-W.C. and P. McAdam contributed to data analysis; all the authors contributed to interpretation of results; L.R. and G.K.A.-A. contributed to the original draft preparation; all the authors contributed to review, editing, and approval of the manuscript.

\section{Data Availability Statement}

All relevant data generated for the biomarker analysis are included in this article and its online supplementary material files. Further inquiries can be directed to the corresponding author.

\section{References}

1 Bai DS, Zhang C, Chen P, Jin SJ, Jiang GQ. The prognostic correlation of AFP level at diagnosis with pathological grade, progression, and survival of patients with hepatocellular carcinoma. Sci Rep. 2017 Oct 9;7(1):12870.

2 Silva JP, Gorman RA, Berger NG, Tsai S, Christians KK, Clarke CN, et al. The prognostic utility of baseline alpha-fetoprotein for hepatocellular carcinoma patients. J Surg Oncol. 2017 Dec;116(7):831-40.

3 Llovet JM, Peña CE, Lathia CD, Shan M, Meinhardt G, Bruix J. Plasma biomarkers as predictors of outcome in patients with ad- vanced hepatocellular carcinoma. Clin Cancer Res. 2012 Apr 15;18(8):2290-300.

4 Shao YY, Hsu CH, Cheng AL. Predictive biomarkers of sorafenib efficacy in advanced hepatocellular carcinoma: are we getting there? World J Gastroenterol. 2015 Sep 28;21(36): 10336-47.

5 Rimassa L, Abbadessa G, Personeni N, Porta C, Borbath I, Daniele B, et al. Tumor and circulating biomarkers in patients with secondline hepatocellular carcinoma from the randomized phase II study with tivantinib. Oncotarget. 2016 Nov 8;7(45):72622-33.
6 Teufel M, Seidel H, Köchert K, Meinhardt G, Finn RS, Llovet JM, et al. Biomarkers associated with response to regorafenib in patients with hepatocellular carcinoma. Gastroenterology. 2019 May;156(6):1731-41.

7 Zhu AX, Kang YK, Yen CJ, Finn RS, Galle PR, Llovet JM, et al. Ramucirumab after sorafenib in patients with advanced hepatocellular carcinoma and increased alpha-fetoprotein concentrations (REACH-2): a randomised, double-blind, placebo-controlled, phase 3 trial. Lancet Oncol. 2019 Feb;20(2):282-96. 
8 Yakes FM, Chen J, Tan J, Yamaguchi K, Shi Y, Yu P, et al. Cabozantinib (XL184), a novel MET and VEGFR2 inhibitor, simultaneously suppresses metastasis, angiogenesis, and tumor growth. Mol Cancer Ther. 2011 Dec; 10(12):2298-308.

9 Abou-Alfa GK, Meyer T, Cheng AL, ElKhoueiry AB, Rimassa L, Ryoo BY, et al. Cabozantinib in patients with advanced and progressing hepatocellular carcinoma. $\mathrm{N}$ Engl J Med. 2018 Jul 5;379(1):54-63.

10 Kelley RK, Meyer T, Rimassa L, Merle P, Park JW, Yau T, et al. Serum alpha-fetoprotein levels and clinical outcomes in the phase III CELESTIAL study of cabozantinib versus placebo in patients with advanced hepatocellular carcinoma. Clin Cancer Res. 2020 Sep 15; 26(18):4795-804.

11 Eisenhauer EA, Therasse P, Bogaerts J, Schwartz LH, Sargent D, Ford R, et al. New response evaluation criteria in solid tumours: revised RECIST guideline (version 1.1). Eur J Cancer. 2009 Jan;45(2):228-47.

12 Johnson PJ, Berhane S, Kagebayashi C, Satomura S, Teng M, Reeves HL, et al. Assessment of liver function in patients with hepatocellular carcinoma: a new evidence-based approach-the ALBI grade. J Clin Oncol. 2015 Feb 20;33(6):550-8.

13 Bruix J, Cheng AL, Meinhardt G, Nakajima K, De Sanctis Y, Llovet J. Prognostic factors and predictors of sorafenib benefit in patients with hepatocellular carcinoma: analysis of two phase III studies. J Hepatol. 2017 Nov; 67(5):999-1008.

14 Meyer T, Kelley RK, Mangeshkar M, Cheng AL, El-Khoueiry AB, Abou-Alfa GK. Prognostic and predictive factors from the phase III CELESTIAL trial of cabozantinib (C) versus placebo $(\mathrm{P})$ in previously treated advanced hepatocellular carcinoma (aHCC). Ann Oncol. 2019;30(Suppl 5):V287-8. Abstract $749 \mathrm{P}$.

15 Shao YY, Huang CC, Lin SD, Hsu CH, Cheng AL. Serum insulin-like growth factor-1 levels predict outcomes of patients with advanced hepatocellular carcinoma receiving antiangiogenic therapy. Clin Cancer Res. 2012 Jul 15;18(14):3992-7.

16 Chan SL, Chan AW, Chan AK, Jian P, Mo F, Chan CM, et al. Systematic evaluation of circulating inflammatory markers for hepatocellular carcinoma. Liver Int. 2017 Feb;37(2): 280-9.

17 Sanghera C, Teh JJ, Pinato DJ. The systemic inflammatory response as a source of biomarkers and therapeutic targets in hepatocellular carcinoma. Liver Int. 2019 Nov;39(11): 2008-23.
18 Zhou L, Liu XD, Sun M, Zhang X, German P, Bai S, et al. Targeting MET and AXL overcomes resistance to sunitinib therapy in renal cell carcinoma. Oncogene. 2016 May;35(21): 2687-97.

19 Pinato DJ, Brown MW, Trousil S, Aboagye $\mathrm{EO}$, Beaumont J, Zhang $\mathrm{H}$, et al. Integrated analysis of multiple receptor tyrosine kinases identifies Axl as a therapeutic target and mediator of resistance to sorafenib in hepatocellular carcinoma. Br J Cancer. 2019 Mar; 120(5):512-21.

20 Murukesh N, Dive C, Jayson GC. Biomarkers of angiogenesis and their role in the development of VEGF inhibitors. Br J Cancer. 2010 Jan 5;102(1):8-18.

21 Leibowitz-Amit R, Pintilie M, Khoja L, Azad $\mathrm{AA}$, Berger R, Laird $\mathrm{AD}$, et al. Changes in plasma biomarkers following treatment with cabozantinib in metastatic castration-resistant prostate cancer: a post hoc analysis of an extension cohort of a phase II trial. J Transl Med. 2016 Jan 13;14:12.

22 Tolaney SM, Ziehr DR, Guo H, Ng MR, Barry WT, Higgins MJ, et al. Phase II and biomarker study of cabozantinib in metastatic triplenegative breast cancer patients. Oncologist. 2017 Jan;22(1):25-32.

23 Winer AG, Motzer RJ, Hakimi AA. Prognostic biomarkers for response to vascular endothelial growth factor-targeted therapy for renal cell carcinoma. Urol Clin North Am. 2016 Feb;43(1):95-104.

24 Yuasa T, Takahashi S, Hatake K, Yonese J, Fukui I. Biomarkers to predict response to sunitinib therapy and prognosis in metastatic renal cell cancer. Cancer Sci. 2011 Nov; 102(11): 1949-57.

25 Ebos JM, Lee CR, Christensen JG, Mutsaers AJ, Kerbel RS. Multiple circulating proangiogenic factors induced by sunitinib malate are tumor-independent and correlate with antitumor efficacy. Proc Natl Acad Sci U S A. 2007 Oct 23; 104(43):17069-74.

26 Chan SL, Mo FK, Johnson PJ, Hui EP, Ma BB, Ho WM, et al. New utility of an old marker: serial alpha-fetoprotein measurement in predicting radiologic response and survival of patients with hepatocellular carcinoma undergoing systemic chemotherapy. J Clin Oncol. 2009 Jan 20;27(3):446-52.

27 Shao YY, Lin ZZ, Hsu C, Shen YC, Hsu CH, Cheng AL. Early alpha-fetoprotein response predicts treatment efficacy of antiangiogenic systemic therapy in patients with advanced hepatocellular carcinoma. Cancer. $2010 \mathrm{Oct}$ 1;116(19):4590-6.
28 Yau T, Yao TJ, Chan P, Wong H, Pang R, Fan ST, et al. The significance of early alpha-fetoprotein level changes in predicting clinical and survival benefits in advanced hepatocellular carcinoma patients receiving sorafenib. Oncologist. 2011;16(9):1270-9.

29 Personeni N, Bozzarelli S, Pressiani T, Rimassa L, Tronconi MC, Sclafani F, et al. Usefulness of alpha-fetoprotein response in patients treated with sorafenib for advanced hepatocellular carcinoma. J Hepatol. 2012 Jul;57(1): 101-7.

30 Chau I, Park JO, Ryoo BY, Yen CJ, Poon R, Pastorelli D, et al. Alpha-fetoprotein kinetics in patients with hepatocellular carcinoma receiving ramucirumab or placebo: an analysis of the phase 3 REACH study. Br J Cancer. 2018 Jul;119(1):19-26.

31 Bruix J, Reig M, Merle P, Kudo M, Meinhardt G, Zhang M, et al. Alpha-fetoprotein (AFP) response in patients with unresectable hepatocellular carcinoma (HCC) in the phase III RESORCE trial. Ann Oncol. 2019;30(Suppl 5):V291. Abstract 755P.

32 Zhu AX, Dayyani F, Yen CJ, Ren Z, Bai Y, Meng Z, et al. Alpha-fetoprotein (AFP) kinetics as a potential surrogate biomarker in patients (pts) with hepatocellular carcinoma (HCC) treated with atezolizumab (atezo) + bevacizumab (bev). ILCA Annual Conference, 2020 Book of Abstracts; 2020. Abstract O-18.

33 Marshall SW. Power for tests of interaction: effect of raising the Type I error rate. Epidemiol Perspect Innov. 2007 Jun 19;4:4.

34 Rimassa L, Assenat E, Peck-Radosavljevic M, Pracht M, Zagonel V, Mathurin P, et al. Tivantinib for second-line treatment of METhigh, advanced hepatocellular carcinoma (METIV-HCC): a final analysis of a phase 3, randomised, placebo-controlled study. Lancet Oncol. 2018 May; 19(5):682-93.

35 Chen Y, Li S, Li W, Yang R, Zhang X, Ye Y, et al. Circulating tumor cells undergoing EMT are poorly correlated with clinical stages or predictive of recurrence in hepatocellular carcinoma. Sci Rep. 2019 May 8;9(1):7084.

36 Lacy S, Yang B, Nielsen J, Miles D, Nguyen L, Hutmacher M. A population pharmacokinetic model of cabozantinib in healthy volunteers and patients with various cancer types. Cancer Chemother Pharmacol. 2018 Jun; 81(6):1071-82.

37 Kelley RK, Oliver JW, Hazra S, Benzaghou F, Yau T, Cheng AL, et al. Cabozantinib in combination with atezolizumab versus sorafenib in treatment-naive advanced hepatocellular carcinoma: COSMIC-312 Phase III study design. Future Oncol. 2020 Jul;16(21):1525-36.
Plasma Biomarkers for Cabozantinib versus Placebo in HCC
Liver Cancer 2022;11:38-47

DOI: $10.1159 / 000519867$ 